\title{
Image Denoising Method Using Diffusion Equation and Edge Map Estimated with K-Means Clustering Algorithm
}

\author{
Woong Hee Kim and Thomas Sikora \\ Communication Systems Group \\ Technical University of Berlin \\ \{whkim, sikora\}@nue.tu-berlin.de
}

\begin{abstract}
The purpose of noise filtering is to preserve features such as edge or corners, and to reduce noise. However, it is difficult to preserve them after noise filtering, because most of noise filtering algorithms have the characteristic of low pass filter, which results in the loss of signal components with high frequencies like edge or corners. Noise filtering methods using diffusion equation try to solve this problem, and improve it to some extent. Edge detector has been used in filtering methods with diffusion equation to determine the speed of diffusion term, so the performance of them depends on the edge detection algorithm to some extent. Previous noise filtering approaches of diffusion equation use a simple gradient edge detector to determine the speed of diffusion. Also, each channel of color images are filtered separately, which result in the filtered image with distortion or blurring. In this paper, we propose a new noise filtering method for color images using diffusion equation and edge map estimated with K-means clustering algorithm together.
\end{abstract}

\section{Introduction}

Many noise filtering algorithms using diffusion equation have been proposed in recent years. They have a diffusion control term such as edge detector, which is based on the gradient of an initial image or successive filtered images by iteration. The diffusion control term decreases the diffusivity near the image features like edge or corners, and increases it in homogeneous region without them. The performance of filtering algorithms depends on the edge detector to some extent. However, the traditional noise filtering algorithms of diffusion equation use a simple edge detection method based on gradient of pixel values. Also, diffusion control term is re-estimated with successive filtered images in every iteration. These kinds of noise filtering algorithms still have a problem of blurring the image features. The dislocation of edge also still remain the problem, because the edge detection used to control diffusivity is made in scale spaces. If each color channel is filtered separately, the filtered image would have the color distortion or blurring. That is because each channel has different level of pixel intensities near the image features such as edge or corners.

In this paper, we present a new color image denoising method, which is based on the edge detector using K-means clustering algorithm and diffusion equation. The edge map for image denoising is evaluated with the initial image, not successive filtered images. We make a different approach, which is filtering an edge map with diffusion equation. All of the color channels of an image have a common diffusion controlling term. We use the filtered edge map as a diffusion control term. This paper is organized as follows. In section 2, the background and related works on noise filtering algorithms using diffusion equation and the fundamentals of K-means clustering are explained. Section 3 presents the proposed noise filtering algorithm. Section 4 shows the experimental results. We compare our method with other noise filtering algorithm using diffusion equation, which we try to improve. Finally, section 5 concludes the paper.

\section{Diffusion Equation for Noise Filtering and K-Means Clustering}

\subsection{Noise Filtering Using Diffusion Equa- tion}

Perona and Malik made an important contribution to noise filtering method, which uses diffusion equation to preserve edge or boundaries [5]. They proposed a new diffusion equation(eq. (1)) with the initial condition eq. (2).

$$
\begin{aligned}
& \frac{\partial u}{\partial t}=\nabla \cdot(g(|\nabla u|) \nabla u) \\
& u(x, y, t=0)=I(x, y)
\end{aligned}
$$


where $I(x, y)$ is a two-dimensional image, which maps $I: \mathbb{R}^{2} \rightarrow \mathbb{R}$.

In eq. (1), $g(s)$ is a nonnegative monotonically decreasing function. For example,

$$
g(s)=e^{\left(-\frac{s^{2}}{K}\right)}
$$

or

$$
g(s)=\frac{1}{1+K s^{2}}
$$

where $K$ is a constant.

However, when we apply this method to reduce noise, image features such as edge or corners are blurred, or noises still remain in the image. Weickert proposed the texture enhancement algorithm using structure tensor information [6]. The diffusivity control term does not depend on the scalar value, but vector quantity in that algorithm. It shows the better performance to enhance texture or coherence, but it still has the same problem. Kim et al proposed a noise filtering algorithm with structure tensor and PCA [3]. It is based on perceptual modeling and shows good results for denoising. However, the computation complexity of it is high, because PCA itself requires high computational complexities. Also, every iteration step in that algorithm requires evaluating the mask in the scale space. Alvarez, Lions, and Morel modified Perona-Malik diffusion equation(eq. (1)) as follows.

$$
\frac{\partial u}{\partial t}=g\left(\left|\nabla G_{\sigma} * u\right|\right)|\nabla u| \nabla \cdot\left(\frac{\nabla u}{|\nabla u|}\right)
$$

where $G_{\sigma}$ is a convolution kernel with kernel size $\sigma$, and * means convolution operation.

Nordström [4] proposed a balancing term or regularization term in Perona-Milik's model(eq. (1)), which is described by eq. (4). The balancing term makes the filtered image close to the initial image.

$$
\frac{\partial u}{\partial t}-\nabla \cdot(g(\nabla u) \nabla u)=I-u
$$

Barcelos et al. [1] proposed a new parabolic equation for image restoration using a balancing term, which results in eq. (5).

$$
\frac{\partial u}{\partial t}=g|\nabla u| \nabla \cdot\left(\frac{\nabla u}{|\nabla u|}\right)-\lambda(1-g)(u-I)
$$

where $g$ means $g\left(\left|\nabla G_{\sigma} * u\right|\right)$, and $\lambda$ is a constant. Also, $g\left(\left|\nabla G_{\sigma} * u\right|\right)$ means that the edge detection is made in Gaussian scale space, and the gradient values are filtered with Gaussian kernel to reduce the effect of noise. The diffusion control term of these diffusion equation is a function of gradient of images in scale space.

\section{$2.2 \quad$ K-Means Clustering}

K-Means clustering algorithm is a kind of unsupervised learning algorithms to cluster given data into a fixed number of groups. Initially, the algorithm groups the data into $k$ partitions, and evaluate the mean or a center point of each partition. After evaluating initial central points, reassign each data point into a group, which has a central point closest to it. The process of reassignment and evaluating central points of each groups is repeated until the metric $V$ in eq. (6) reaches minimum [2].

$$
V=\sum_{i=1}^{k} \sum_{x_{j} \in S_{i}} d\left(x_{j}, c_{i}\right)
$$

where $k$ means a fixed number of clusters in data set $S_{i}, c_{i}$ represents the center point or mean point of all of the data $x_{j}$ in $S_{i}$, and $d(x, c)$ is a chosen distance measure or function. A typical distance function is a squared error or $d(x, c)=$ $|x-c|^{2}$. The result of K-Means clustering algorithm always has $k$ groups, and each group has one object at least.

\section{Noise Reduction Method with Filtered Edge Map}

Most of edge detection method in diffusion equation, which controls the speed of diffusion, uses the gradient information in scale spaces. That kind of edge detector is dependent on the scale size, and may have a problem of edge dislocation. To reduce this kind of problem, we extract the information of controlling diffusion speed from an initial image. However, if the initial image is noisy, it is difficult to determine that a pixel belongs to edge or not. We assume that noise is additive and independent from signal as shown in eq. (7).

$$
\mathscr{I}(x, y)=I_{o}(x, y)+\mathscr{N}(x, y)
$$

where $\mathscr{I}(x, y)$ is a noisy image data, $I_{o}(x, y)$ denotes the ideal image data without noise, and $\mathscr{N}(x, y)$ represents the signal independent noise. Also, we assume that there are two regions in a set of pixel values from a small fixed size window such as $3 \times 3$ window.

First, we cluster pixel values within a fixed window into two groups with K-Means clustering algorithm. We model the difference between the center of each group as edge strength as follows.

$$
E S(i, j)=\left|c_{1}(i, j)-c_{2}(i, j)\right|
$$

where $c_{1}(i, j)$ represents the center of the first group, and $c_{2}(i, j)$ is the center of the second group. Fig. 2 depicts the edge strength of Fig. 1. After evaluating initial edge map, 


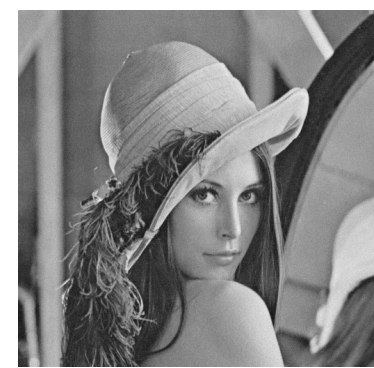

Figure 1. Initial image of Lena with noise standard deviation 5

we apply noise filtering technique of eq. (5) to the initial edge map, which results in the filtered edge map.

$$
\frac{\partial v}{\partial t}=g|\nabla v| \nabla \cdot\left(\frac{\nabla v}{|\nabla v|}\right)-\lambda(1-g)(v-E)
$$

whre $E$ means the initial edge map evaluated with eq. (8). Edge map has better characteristic to apply noise filtering based on diffusion equation, because the difference of values in edge is large, and it is smooth in a homogeneous region. In other words, the values in homogeneous regions, which do not include image features, change smoothly, and those in edge region change abruptly. That is a better condition for noise filtering based on diffusion equation. Therefore, filtering edge map produces better result than applying filtering to an image directly.

Finally, we use the extracted edge information from the initial image as a parameter of function of $g$ in eq. (5) instead of the magnitude of the smoothed gradient with Gaussian filtering.

$$
\frac{\partial u}{\partial t}=v|\nabla u| \nabla \cdot\left(\frac{\nabla u}{|\nabla u|}\right)-\lambda(1-v)(u-I)
$$

where $v=g(E S)=e^{\left(-\frac{E S^{2}}{K}\right)}$. As we mentioned before, the diffusion control term is evaluated in the beginning of filtering with an initial image once, which means that the evaluation of diffusion control term is not required in every iteration step. This edge map is used as the common diffusion controlling term for each color channel of the color image.

\section{Experimental Results}

In this section, we present results from experiment with our proposed method. Diffusion term in eq. (9) is calculated as follows [1].

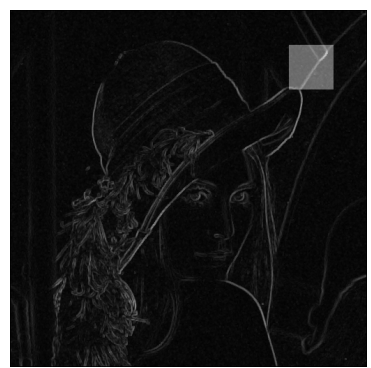

(a)

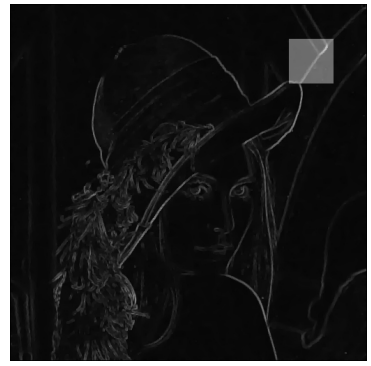

(c)

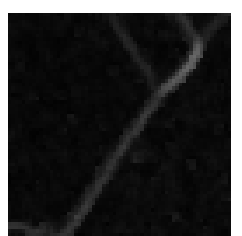

(b)

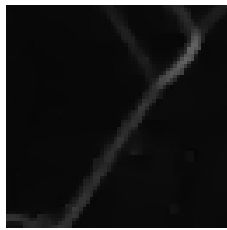

(d)
Figure 2. Initial edge map and filtered edge map of Fig. 1. (a) initial edge map, (b) highlighted region of (a), (c) filtered edge map, (d) highlighted region of (b)

$|\nabla u|\left(\nabla \cdot\left(\frac{\nabla u}{|\nabla u|}\right)\right)=\frac{u_{x}{ }^{2} u_{y y}-2 u_{x} u_{y} u_{x y}+u_{y}{ }^{2} u_{x x}}{u_{x}{ }^{2}+u_{y}{ }^{2}}$

where $u_{x}$ represents a partial derivative of $u$ with respect to $x$. Eq. (10) is approximation of a diffusion term with central difference and Euler's method. We use a fixed size window for K-Means clustering, which has a size $3 \times 3$.

To compare the performance of the proposed algorithm, we evaluate PSNR (Peak Signal-to-Noise Ratio) of Lena images with noise. Table 1 shows PSNR of them, where Lena 1, Lena 2, and Lena 3 mean a Lena image with Gaussian noise standard deviation 5, 10, and 20 respectively. Our proposed algorithm shows better results in the point of PSNR as shown in Table 1.

Table 1. Comparison of PSNR(dB)

\begin{tabular}{|c|c|c|c|}
\hline & Lena 1 & Lena 2 & Lena 3 \\
\hline \hline Proposed Method & 37.50 & 33.38 & 26.83 \\
\hline Barcelos's Method & 36.82 & 32.38 & 24.58 \\
\hline
\end{tabular}

We also apply our proposed method to an image taken with a digital camera. The model of the digital camera used in the experiment is Canon EOS 300D, and ISO setting value of it is 800 . If we increase ISO value in a digital 


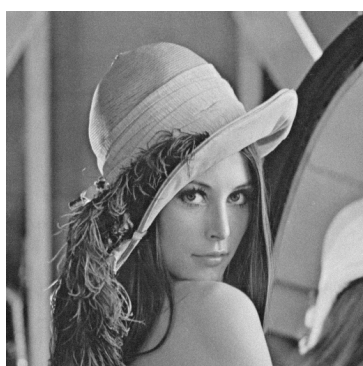

(a)

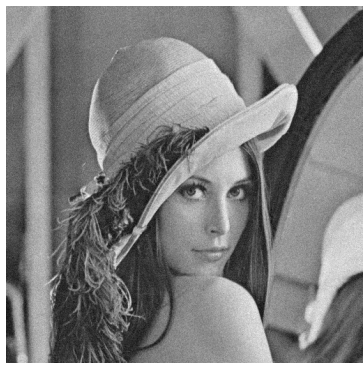

(c)

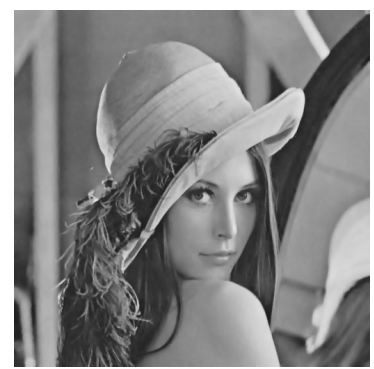

(b)

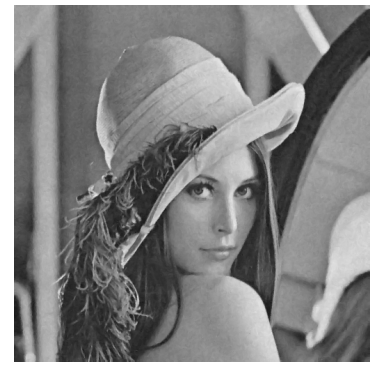

(d)
Figure 3. Filtering results. (a) Lena image with noise standard deviation 5 , (b) filtered image of (a), (c) Lena image with noise standard deviation 10, (d) filtered image of (c)

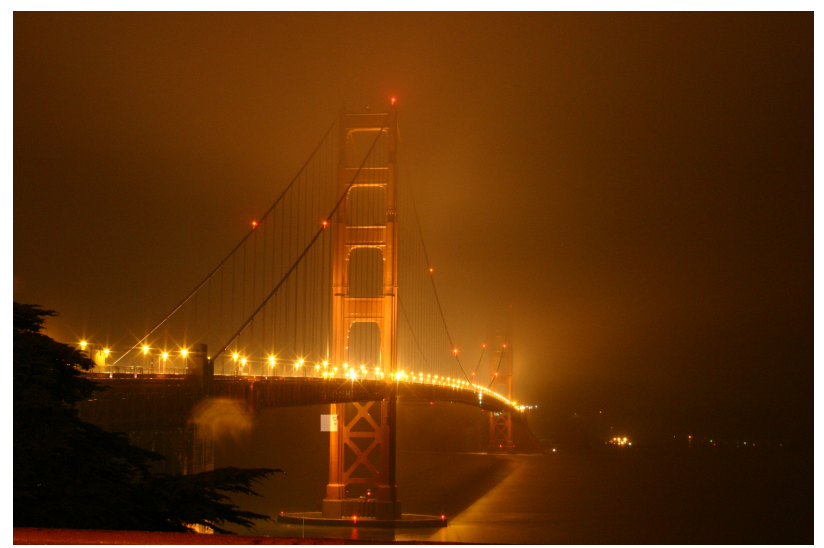

(a)

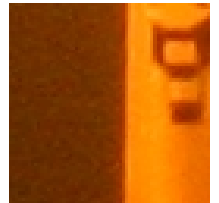

(b)

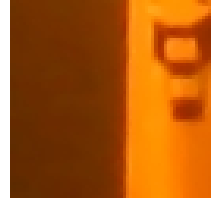

(c)
Figure 4. Image with significant ISO noise from a digital camera. (b) is the highlighted part of (a) before filtering, and (c) shows the same region as (b) after filtering camera higher and higher, the picture becomes more and more noisy due to high ISO noise. Fig. 4 shows a test image with significant ISO noise. Fig. 4(b) is the region of the highlighted part in Fig. 4(a). As shown in Fig. 4(c), our noise filtering algorithm preserves image features such as edge or corners, and reduces noise well.

\section{Conclusion}

In this paper, we have presented a new noise filtering method using edge map estimated with diffusion equation and K-Means clustering algorithm together. We make a different approach to evaluate diffusion control term. We use a filtered edge map as a diffusion control term. We refine the edge map with diffusion equation after clustering pixel values within a window into two groups. Edge information to control diffusion speed is extracted from the initial image, not from scale spaces to improve the problem of edge dislocation and blurring of image features. Also, the edge map has better characteristic for diffusion based noise filtering, and we make a use of it. The edge map is used as the common diffusion controlling term for noise filtering of a color image. Experimental results shows that our proposed method preserves edge and reduces noise well and better results in the point of image quality.

\section{ACKNOWLEDGMENT}

The work presented was developed within VISNET2, a European Network of Excellence (http://www.visnetnoe.org), funded under the European Commission IST FP6 programme.

\section{References}

[1] C. A. Z. Barcelos, M. Boaventura, and J. Evanivaldo Castro Silva. A well-balanced flow equation for noise removal and edge detection. IEEE Transactions on Image Processing, 12(7):751-763, July 2003.

[2] S. Haykin. Neural Networks : A Comprehensive Foundation. Prentice Hall, 1998.

[3] J.-H. Kim and T. Sikora. Color image noise reduction using perceptual maximum variation modeling for color diffusion. In 7th International Workshop on Image Analysis for Multimedia Interactive Services(WIAMIS 2006), 2006.

[4] K. Nordström. Biased anisotropic diffusion - a unified regularization and diffusion approach to edge detection. Image Vis. Comput., (8):318-327, 1990.

[5] F. Perona and J. Malik. Scale-space and edge detection using anisotropic diffusion. IEEE Transactions on Pattern Analysis and Machine Intelligence, 12(7):629-639, July 1990.

[6] J. Weickert. Multiscale texture enhancement. In Computer Analysis of Images and Patterns: LNCS in Computer Science, volume 970, pages 230-237, Berlin, 1995. Springer. 